\title{
Benefits of a grape-rich diet on human health: a nutrigenomics study underlining the potential role of non-coding RNAs
}

Rosa Anna Milella1, Marica Gasparro1, Fiammetta Alagna1,2, Maria Francesca Cardone1, Silvia Rotunno1,3 Concetta Tiziana Ammollo4, Fabrizio Semeraro4, Apollonia Tullo5, Flaviana Marzano5, Domenico Catalano ${ }^{6}$, Mario Colucci ${ }^{4}$, Domenica D’Elia ${ }^{6 \bowtie}$

${ }^{1}$ Research Centre for Viticulture and Enology, Council for Agricultural Research and Economics, Turi, Bari, Italy

2ENEA Italian National Agency for New Technologies Energy and Sustainable Economic Development, Trisaia Research Center, Rotondella, Matera, Italy

${ }^{3}$ Institute for Sustainable Plant Protection, National Research Council, Torino, Italy

${ }^{4}$ Department of Biomedical Sciences and Human Oncology, University of Bari "Aldo Moro", Bari, Italy

${ }^{5}$ Institute of Biomembranes, Bioenergetics and Molecular Biotechnologies, National Research Council, Bari

${ }^{6}$ Institute for Biomedical Technologies, National Research Council, Bari, Italy

Competing interests: RAM none; MG none; FA none; MFC none; SR none; CTA none; FS none; AT none; FM none; DC none; MC none; DD none

Grape (Vitis vinifera L.) is one of the most typical fruit of the Mediterranean diet, characterised by high polyphenols content with marked antioxidant and antiinflammatory activities (Goszcz et al., 2017). By using transcriptional profiling techniques, it has become increasingly clear that polyphenols can influence the expression of genes. Many of these genes are key elements of cell signalling cascades (Spencer 2009; Fraga and Oteiza 2011) and regulatory non-coding RNAs (ncRNAs) (Budisan et al., 2017).

A previous study showed that table grape extracts exert a marked antithrombotic activity in vitro (Ammollo et al., 2017). To shed light on the molecular basis of grape intake effects on human health and investigate the potential role of ncRNAs, we carried out a nutrigenomics study (Milella et al., 2020a and 2020b). To this aim, 20 healthy subjects were enrolled to follow a grape-rich diet for 21 days. The gene expression profiles of peripheral blood mononuclear cells (PBMCs) extracted from six of these subjects, after 21 days of fresh table grape-rich diet and after an additional 28-day washout, were analysed. The results showed 930 genes differentially expressed. Among these genes, more than 200 are long ncRNAs (lncRNAs), almost all downregulated after the washout period when polyphenols' direct effect is supposed to be completely exhausted.

The functional analysis of differentially expressed genes revealed significant changes in processes critical for organismal and cell wellbeing such as inflammation and immunity, thrombosis, DNA and protein repair, autophagy and mitochondrial biogenesis. From our analysis of lncRNAs down-regulated in our study, we observed that many of them are over-expressed in many types of tumours, chronic diseases due to the persistency of inflammation and metabolic syndromes such as obesity. Altogether, these findings provide exciting clues for the crucial role of ncRNAs in grape intake's long-term effects on a series of biological processes. These lncRNAs would deserve to be further investigated for potential applications in the care of chronic diseases and cancer and their prevention.

\section{Acknowledgements}

This work was supported by a grant from MIUR (Ministero Italiano dell'Università e della Ricerca project "PON02_00186_2937475 - PROALIFUN") and by the CNR (National Research Council) Flagship Project InterOmics.

\section{References}

1. Ammollo CT et al. (2017) Grape intake reduces thrombin generation and enhances plasma fibrinolysis. Potential role of circulating procoagulant microparticles. J Nutr Biochem 50:6673. http://dx.doi.org/10.1016/j.jnutbio.2017.08.012

2. Budisan L et al. (2017) Dietary Intervention by Phytochemicals and Their Role in Modulating Coding and Non-Coding Genes in Cancer. Int J Mol Sci 18(6). http://dx.doi.org/10.3390/! ijms18061178

3. Fraga CG, Oteiza PI (2011) Dietary flavonoids: Role of (-)-epicatechin and related procyanidins in cell signalling. Free Radic Biol Med 51(4):813-23. http://dx.doi.org/10.1016/j. freeradbiomed.2011.06.002

4. Goszcz K, Duthie GG, Stewart D, Leslie SJ, Megson IL (2017) Bioactive polyphenols and cardiovascular disease: chemical antagonists, pharmacological agents or xenobiotics that drive an adaptive response? Br J Pharmacol 174:1209-25. http://dx.doi. org/10.1111/bph.13708

5. Milella RA et al. (2020b) Gene expression signature induced by grape intake in healthy subjects reveals wide-spread beneficial effects on peripheral blood mononuclear cells. Journal of Functional Food, 64:103705. http://dx.doi.org/10.1016/j. jff.2019.103705

6. Milella RA et al. (2020a) Microarray data and pathway analyses of peripheral blood mononuclear cells from healthy subjects after a three weeks grape-rich diet. Data in Brief 29, 105278. http:/!. dx.doi.org/10.1016/j.dib.2020.105278

7. Spencer JP (2009) Flavonoids and brain health: multiple effects underpinned by common mechanisms. Genes Nutr. 4(4):243-50. http://dx.doi.org/10.1007/s12263-009-0136-3. 